\title{
Correção de cifose secundária à mielomeningocele através de osteotomia vertebral
}

\author{
Spinal osteotomy for kyphosis correction in myelomeningocele
}

Luciano Miller Reis Rodrigues', Edgar Santiago Valesin Filho', Adriano Masayuki Yonezaki', Fabricio Hidetoshi Ueno', Rodrigo Junqueira Nicolau', Luciano Borges Cabral Temporal', Carlo Milani'

\section{Resumo}

Introdução: A mielomeningocele é uma afecção que com frequência evolui com deformidade vertebral, sendo a cifose secundária à mielomeningocele uma manifestação grave e que compromete a qualidade de vida do paciente. Há dificuldade para manter o tronco equilibrado 0 exige apoio das mãos; além disso, há lesões de pele recorrentes no ápice da deformidade e dificuldade para adaptação em cadeiras de rodas. Portanto, a correção cirúrgica da deformidade é necessária. 0 tratamento cirúrgico possui uma ampla variedade de técnicas descritas. Relato do caso: Realizou-se neste estudo uma osteotomia vertebral através de acesso único posterior, associada à vertebrectomia com fixação pedicular e haste de ancoragem distal no forame sacral em uma criança de 10 anos de idade portadora de mielomeningocele de nível torácico. Obteve-se correção significativa da deformidade inicial, com regressão de 110 para menos de $15^{\circ} \mathrm{Cobb}$, com melhora significativa do aspecto funcional e estético, reestabelecimento de tronco ereto e independência para utilização das mãos por parte da paciente para práticas de atividade de vida adaptadas. Discussão: A osteotomia vertebral através de acesso único posterior, associada à vertebrectomia e artrodese ampla para correção de cifose secundária à mielomeningocele, é um procedimento tecnicamente complexo, porém é uma possibilidade viável para pacientes com grave comprometimento vertebral. Conclusão: A correção através da osteotomia vertebral se mostrou eficaz na correção da deformidade para o reequilíbrio da coluna vertebral.

Palavras-chave: Meningomielocele; cifose; curvaturas da coluna vertebral; osteotomia; fusão vertebral.

\begin{abstract}
Introduction: Myelomeningocele is a condition that often evolves with vertebral deformity; the secondary kyphosis that follows from myelomeningocele is a serious condition and compromises the patient's quality of life. There is difficulty to maintain the torso balance requires hand support; there are also recurrent skin lesions when the deformity is aggravated, and a difficulty of adaption to wheelchairs. Therefore, surgical correction of the deformity is required. There is a wide variety of described techniques for surgical treatment. Case report: In this study we carried out a vertebral osteotomy through the unique posterior access associated to vertebrectomy with pedicular fixation and distal anchorage implant on the sacral foramen of a 10-year-old child with myelomeningocele of toracic level. We have obtained significant correction of the initial deformity with decrease from 110 to less than $15^{\circ}$ Cobb, with sensible improvement of functional and aesthetic aspects, reestablishment of the erected torso and independence of the patient's hands for adapted daily practices. Discussion: Vertebral osteotomy through unique posterior access associated to vertebrectomy and arthrodesis for correction of kyphosis following from myelomeningocele is a technically complex procedure; nevertheless it is a viable possibility for patients with a serious vertebral condition. Conclusion: Vertebral osteotomy has shown to be efficient in vertebral deformity correction, restoring equilibrium.
\end{abstract}

Keywords: Meningomyelocele; kyphosis; spinal curvatures; osteotomy; spinal fusion.

Recebido: $16 / 9 / 2008$

Revisado: 16/6/2009

Aprovado: 1/9/2009

Disciplina do Aparelho Locomotor da Faculdade de Medicina do ABC (FMABC). Santo André (SP). Brasi

Endereço para correspondência: Luciano Miller Reis Rodrigues - Centro de Estudos da Disciplina de Doenças do Aparelho Locomotor da FMABC Avenida Henrique Calderazzo, 321 - Vila Assunção - CEP 09190-610 - Santo André (SP) - Fone: (11) 2829-5191 - E-mail: luciano.miller@uol.com.br 


\section{Introdução}

A mielomeningocele é a apresentação mais grave dos disrafismos espinhais, condições nas quais existem anomalias do tecido nervoso e alterações vertebrais ósseas secundárias. Diversas deformidades ocorrem nesses pacientes, sendo as mais frequentes as luxações nos quadris e as deformidades nos joelhos e pés ${ }^{1-3}$. Na coluna vertebral, a cifose congênita pode ser evidente no parto; porém, a angulação posterior rígida da coluna é geralmente notada na infância e limitada ao sítio do defeito ósseo, ocorrendo geralmente nos pacientes com defeitos de fechamento torácico, toracolombar e lombar proximal ${ }^{1-2}$. A deformidade cifótica ocasionada pela falha nas estruturas posteriores de sustentação vertebral e desequilíbrio neuromuscular aumenta progressivamente com o crescimento da criança, geralmente se tornando acentuada em torno de 7 anos de vida ${ }^{3}$.

O agravamento da cifose torna difícil a adaptação à cadeira de rodas e à perda do equilíbrio do tronco, levando a criança a apoiar as mãos para se equilibrar, limitando as atividades de vida diária ${ }^{2-7}$; nesses casos, a correção cirúrgica da deformidade se torna necessária.

\section{Relato do caso}

Relata-se aqui um caso de correção de deformidade cifótica em uma criança de 10 anos através de osteotomia vertebral em cunha de subtração posterior e vertebrectomia associada à artrodese (fusão óssea) com instrumentação. A paciente, de 10 anos e 11 meses, natural e procedente de Santo André, é portadora de mielomeningocele nível torácico, sem função motora abaixo do nível do nível de lesão. A coluna vertebral apresentava deformidade cifótica rígida na transição toracolombar. Funcionalmente, a paciente se encontrava com dificuldade de adaptação à cadeira de rodas devido ao ápice da cifose e à perda do equilíbrio do tronco, sendo necessário o uso das mãos para conseguir se equilibrar (Figura 1).

Na radiografia em perfil na posição sentada, evidenciou-se a deformidade cifótica na transição toracolombar de T11 até L2, de 110 graus Cobb (Figura 2A). Foi solicitada a tomografia com reconstrução 3D para melhor avaliação da deformidade (Figura $2 \mathrm{~B}$ ).

A correção da deformidade foi realizada através da osteotomia de fechamento de base dorsal, através de acesso posterior, com vertebrectomia de T12 e L1 associadas com artrodese desde T4 a S1 (fixação pedicular T4, T5, T6, T7) e fixação distal com haste angulada ancorada no forame sacral (Figura 3).

A abordagem cirúrgica foi realizada por via posterior, com uma incisão cirúrgica longitudinal iniciando na região torácica proximal até o nível de S2, com pequenos desvios na região da cicatriz de fechamento da mielomeningocele, evitando-se a região de pele menos vascularizada. Durante a dissecção por planos foi identificada a dura-máter e rebatido o saco dural não-funcional (Figura 4), ligando e seccionando

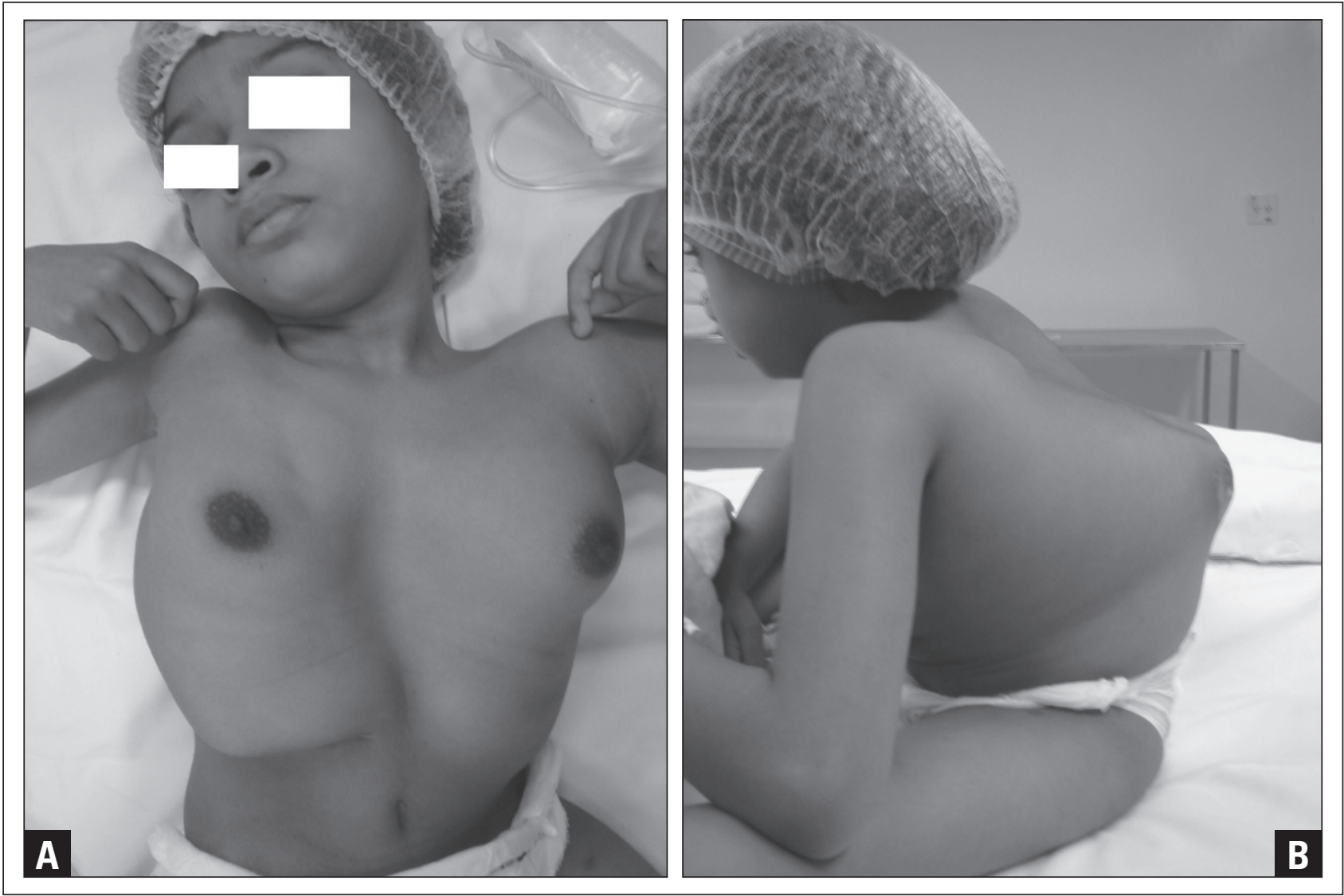

Figura 1 - Deformidade do tronco vista de frente (A) e em perfil (B) 

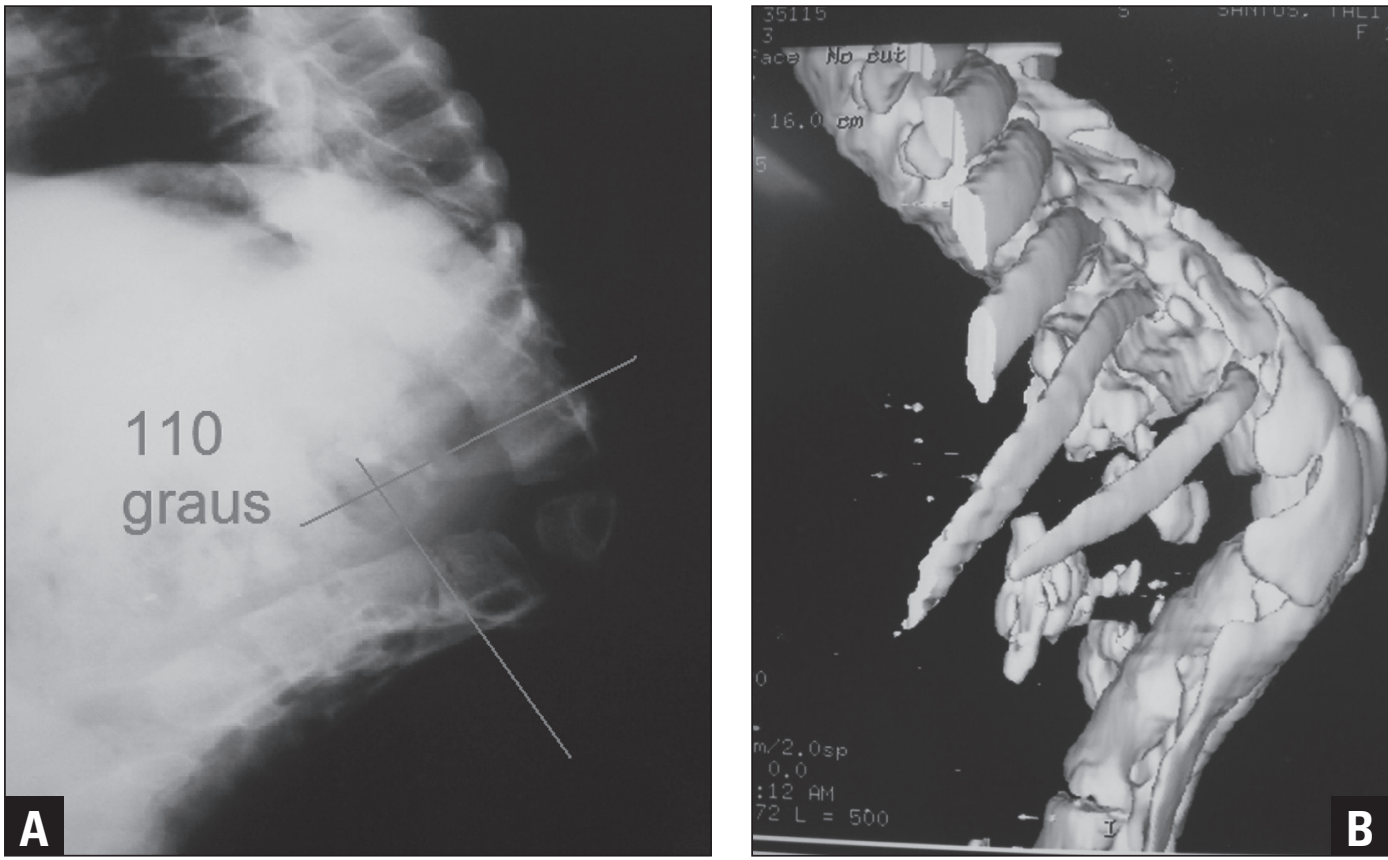

Figura 2 - (A) Radiografia em perfil. (B) Tomografia com reconstrução 3D

as raízes e seus respectivos vasos proximais a região foraminal, com exposição do canal vertebral desde a vértebra apical a ser removida até o nível de S2.

Foi realizada a ressecção de dois corpos vertebrais no ápice da deformidade com a preservação do ligamento longitudinal anterior. A instrumentação foi realizada com duas hastes longitudinais de $5 \mathrm{~mm}$ moldadas em 'S' distal com a introdução da porção terminal da haste no forame de S1, proporcionando uma alavanca com a fixação das vértebras distais para proximal chamada 'manobra de Cantilever'. A instrumentação cirúrgica foi realizada com parafusos pediculares, e a extensão da artrodese foi do segmento T4 a S1.

Foi realizada a decorticação da lâmina e apófises articulares no segmento normal, e os processos transversos e apófises articulares remanescentes nas vértebras com espinha bífida. Foi aplicado enxerto ósseo nos espaços discais, com a realização concomitante de uma artrodese intersomática.

A radiografia pós-operatória demonstrou uma cifose remanescente de $15^{\circ} \mathrm{Cobb}$ (Figura 3). Não houve complicações significativas no pós-operatório. O seguimento da paciente durou três anos sem sinal de soltura, falha da artrodese ou recidiva de deformidade.

\section{Discussão}

A incidência relatada na literatura de mielomeningocele associada com cifose significativa ao nascimento varia de 8 a $20 \%{ }^{7-9}$. O local mais comum de cifose nessa situação ocorre entre T10 e L2 $2^{2}$. Na evolução da doença, a deformidade tende a aumentar à medida que o pacien-

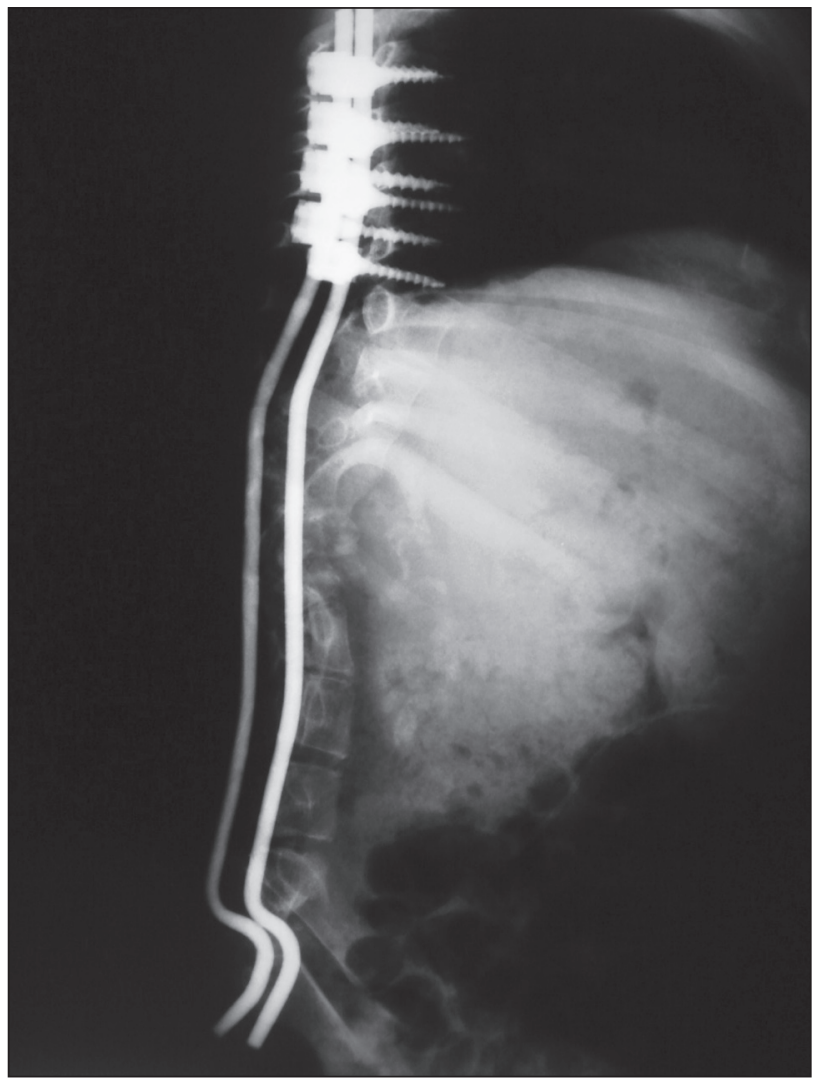

Figura 3 - Aspecto radiográfico pós-operatório

te cresce, postura supina do tronco e aumento de peso; qualquer aumento de deformidade pode ser associado à deterioração neurológica clinicamente apresentada por paralisia de membros inferiores e bexiga 


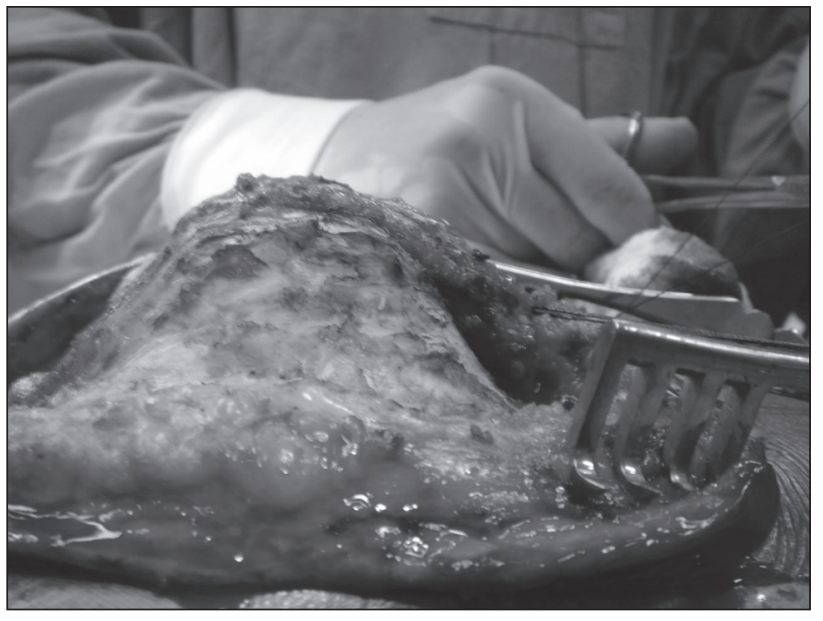

Figura 4 - Isolamento do saco

neurogênica ${ }^{8,10}$. A idade para correção cirúrgica da deformidade é assunto controverso, e a melhor idade para correção cirúrgica da deformidade permanece incerta. As variáveis conflitantes são grau e extensão da deformidade, técnica de estabilização e manutenção do potencial de crescimento do tronco. Huang e colaboradores recomendam postergar a cirurgia até a idade de 10 a 12 anos em crianças que não apresentam lesões de pele no ápice da deformidade e déficit neurológico progressivo $^{6}$. Cirurgia mais precoce é recomendada para crianças com lesões cutâneas recorrentes, alterações posturais graves e comprometimento de função respiratória. Segundo Lindseth ${ }^{7}$, o tratamento da cifose nessas situações deve procurar alcançar três objetivos principais: corrigir e estabilizar a coluna para permitir equilíbrio sentado, diminuir a proeminência óssea e permitir um aumento da altura do tronco em sua região abdominal. A fusão é necessária para manutenção da correção da deformidade; porém, procedimentos extensos em crianças pequenas podem ser problemáticos, devido a um crescimento desproporcional do tronco.

São considerados pré-requisitos para tratamento cirúrgico de cifose em portadores de mielomeningocele: boas condições de pele na área de cifose; patência de derivação liquórica e ausência de infecção urinária ativa ${ }^{6}$. Kim e colaboradores ${ }^{11}$ enumeram os principais fatores de risco para complicações: idade avançada no tempo da correção, artrodese anterior e posterior combinadas, deformidades graves e déficit neurológico preexistente.

O uso de órteses em deformidades rígidas, além de não corrigir a deformidade, não contém a sua progressão, sendo útil apenas em tratamento de curvas vertebrais compensatórias secundárias ${ }^{2,3}$. Existe uma discussão na literatura quanto à artrodese posterior ou combinada durante a correção cirúrgica, sendo a artrodese posterior isolada geralmente mais indicada para crianças novas e deformidades com menos de $55^{\circ} \mathrm{Cobb}$; e artrodeses combinadas para crianças maiores, diminuindo a taxa de pseudartrose e perda da correção, como realizado no caso relatado ${ }^{11,12}$.
A osteotomia vertebral e a vertebrectomia (ressecção de segmento único ou múltiplo) podem ser procedimentos necessários e complementares para se conseguir maior grau de correção e evitar tração nas estruturas anteriores, como a aorta, veia cava e parede abdominal ${ }^{5}$. Neste estudo, a osteotomia realizada incluiu duas vértebras e houve remoção completa do componente discal no bloco ressecado, ampliando a grau de correção angular através de uma cunha em apenas dois níveis vertebrais.

A área de fusão e os níveis de instrumentação dependem do grau de flexibilidade da curva compensatória da deformidade no plano sagital e devem ser decididos levando em consideração cada paciente e sua deformidade ${ }^{4}$. Na técnica descrita por Heydemann e Gillespie e aplicada por Huang ${ }^{6}$, é necessária a divisão do saco dural e da medula em segmentos proximais e distais à osteotomia; na técnica utilizada neste relato, utilizou-se a dissecção circunferencial do saco dural sem transecção, permitindo assim sua ampla mobilização para realizar a osteotomia e evitando sangramento excessivo e perda liquórica, complicação frequente na secção dos elementos neurais.

Entretanto, alguns autores não contraindicam a divisão do saco dural; segundo Sharrard ${ }^{10}$, quando houver paralisia completa de função vesical e membros inferiores, a osteotomia transversa da cifose pode ser realizada. A técnica recomenda dissecção das estruturas neurais paralisadas e fechamento da dura-máter no nível superior da cifose. Utilizou-se a fixação distal das hastes no forame sacral, visando a uma ancoragem mais estável e a menores riscos de recorrência de deformidade. Essa tem sido uma tendência na literatura, uma vez que a fixação no ilíaco é precária devido à osteoporose e displasia da espinha e crista ilíaca nesses pacientes ${ }^{5}$. Sharrard ${ }^{10}$ demonstrou uma melhora angular média após procedimento cirúrgico para correção de deformidade de $33^{\circ} \mathrm{em}$ crianças maiores de 5 anos; entretanto, houve uma média de perda de correção de $11^{\circ} \mathrm{em}$ dois anos. A literatura aponta para uma recidiva em graus variáveis da deformidade após tratamento cirúrgico, porém a cirurgia diminui a taxa de progressão que seria observada na história natural da doença. Neste caso, obteve-se uma melhora de aproximadamente $90^{\circ}$ na deformidade, compatível com relatos da literatura ${ }^{1,4,7}$. Não existe técnica padronizada para tratamento cirúrgico de deformidades graves em cifose congênita, devido à variedade de etiologias e apresentações clínicas ${ }^{1}$. Os resultados de procedimentos envolvendo correção de cifose secundária à mielomeningocele permanecem muito distantes do ideal, e muito precoces para previsão de resultados futuros ${ }^{10}$. Existe um grande desafio no tratamento da cifose na mielomenigocele devido à necessidade de procedimentos cirúrgicos muitas vezes extensos em pacientes com alterações de pele, baixo peso e bexiga neurogênica.

A correção através da osteotomia vertebral se mostrou eficaz na correção da deformidade para o reequilíbrio da coluna vertebral. 


\section{Referências}

1. Smith JT, Gollogly S, Dunn HK. Simultaneous anterior-posterior approach through a costotransversectomy for the treatment of congenital kyphosis in acquired kyphoscoliotic deformities. J Bone Joint Surg Am 2005;87(10): 2281-9

2. Tachdjian MO. Ortopedia pediátrica: diagnóstico e tratamento. 1a ed. Rio de Janeiro: Revinter; 2001.

3. Lindseth RE. Mielomeningocele. In: Morrissy RT, Weinstein SL, editors. Ortopedia pediátrica de Lovell e Winter. 5a ed. São Paulo: Manole; 2005.

4. Domanic U, Talu U, Dikici F, Hamzaoglu A. Surgical correction of kyphosis: posterior total wedge resection osteotomy in 32 patients. Acta Orthop Scand 2004;75(4):449-55.

5. Heydemann JS, Gillespie R. Management of myelomeningocele kyphosis in the older child by kyphectomy and segmental spine instrumentation. Spine (Phila Pa 1976) 1987;12(1):37-41.
6. Huang TJ, Lubicky JP. Kyphectomy and segmental spine instrumentation in young children with myelomeningocele kyphosis. J Formos Med Assoc 1994;93(6):503-8.

7. Lindseth RE, Stelzer L. Vertebral excision in children with myelomeningocele. J Bone Joint Surg Am 1979;61(5):699-704.

8. Hoppenfeld S. Congenital kyphosis in myelomeningocele. J Bone Joint Surg $\mathrm{Br} 1967 ; 49(2): 276-82$.

9. Sharrard WJW. Spinal osteotomy for congenital kyphosis in myelomeningocele. J Bone and Joint Surg 1968;50(3):466-71.

10. Kim YJ, Otsuka NY, Flynn JM, Hall JE, Emans JB, Hresko MT. Surgical treatment of congenital kyphosis. Spine (Phila Pa 1976) 2001;26(20):2251-7.

11. McMaster MJ, Singh H. Surgical management of congenital kyphosis and kyphoscoliosis. Spine (Phila Pa 1976) 2001;26(19):2146-55. 